\title{
Analysis of EEG signal to detect motor command generation towards stroke rehabilitation
}

Book or Report Section

Accepted Version

Hayashi, Y., Nagai, K., Ito, K., Nasuto, S. J., Loureiro, R. C. V. and Harwin, W. S. (2013) Analysis of EEG signal to detect motor command generation towards stroke rehabilitation. In: Pons, J. L., Torricelli, D. and Pajaro, M. (eds.) Converging Clinical and Engineering Research on Neurorehabilitation. Biosystems and Biorobotics (1). Springer, pp. 569-573. ISBN 9783642345456 doi: https://doi.org/10.1007/978-3-642-345463_92 (Proceedings of ICNR 2012, the International Conference on NeuroRehabilitation) Available at http://centaur.reading.ac.uk/31273/

It is advisable to refer to the publisher's version if you intend to cite from the work. See Guidance on citing.

To link to this article DOI: http://dx.doi.org/10.1007/978-3-642-34546-3_92

Publisher: Springer 
All outputs in CentAUR are protected by Intellectual Property Rights law, including copyright law. Copyright and IPR is retained by the creators or other copyright holders. Terms and conditions for use of this material are defined in the End User Agreement.

\section{www.reading.ac.uk/centaur}

\section{CentAUR}

Central Archive at the University of Reading

Reading's research outputs online 


\title{
Analysis of EEG Signal to Detect Motor Command Generation Towards Stroke Rehabilitation
}

\author{
Yoshikatsu Hayashi, Kiyoshi Nagai, Koji Ito, Slawomir J. Nasuto, Rui C.V. Loureiro and William S. Harwin
}

\begin{abstract}
Our aim is to reconstruct the brain-body loop of stroke patients via an EEG-driven robotic system. After the detection of motor command generation, the robotic arm should assist patient's movement at the correct moment and in a natural way. In this study we performed EEG measurements from healthy subjects performing discrete spontaneous motion. An EEG analysis based on the temporal correlation of the brain activity was employed to determine the onset of single motion motor command generation..
\end{abstract}

\section{INTRODUCTION}

Recent work has shown that motor rehabilitation during the acute stages can decrease the effect of stroke. It is important to consider motor learning in the context of brain plasticity. The signal flow in a motor control system can be described as: a motor command, generated in the motor area, that goes through the spinal cord and finally activates specific muscles. After muscle contaction, sensory feedback is transmitted to the somatosensory area in the cortex. This flow makes up the sensory-motor closed loop. However, stroke patients have difficulty learning specific motions because the loop is damaged. If a correlation between the motor command generation and the feedback signal to the somatosensory cortex can be achieved, this opens up the possibility for simulating the sensory-motor closed loop and thus enhance motor learning [1].

On the other hand, new rehabilitation techniques have emerged such as constraint-induced therapy, biofeedback therapy and robot-aided therapy [2]. In particular robotic techniques allow precise recording of movements and application of forces to the affected limb, making it a valuable tool for motor rehabilitation. In our previous study [3] we reported the development of an assistive robotic arm with a magnetic levitation joint which applies compliant motion to the subject's upper limb.

Our general aim is to reconstruct the brain-body loop for stroke patients. In order to facilitate the reconstruction of the closed loop between brain and body via the EEG-driven robotic system, the appropriate assistance should be applied to patients at the right moment after the moment of motor

This work was supported by The Ministry of Education, Culture, Sports, Science and Technology of Japan, Grant-in-Aid for Scientific Research (B), No. 24300206 and by Grant-in-Aid for Research Promotion, Ritsumeikan University.

Y. Hayashi, K. Nagai and K. Ito are with Department of Robotics, College of Science and Engineering, Ritsumeikan University, 1-1-1 Noji higashi, Kusatsu-shi, Shiga 525-8577, Japan haya efc.ritsumei.ac.jp

S.J. Nasuto and W.S. Harwin are with School of Systems Engineering, University of Reading, Reading RG6 6AY, United Kingdom

R.C.V. Loureiro is with School of Engineering and Information Sciences, Middlesex University, London, NW4 4BT, United Kingdom command generation so that the motor command and the feedback signal to the somatic sensory area in the brain can be linked in the closed loop. To determine the moment of motor command generation in a single trial with respect to the onset of motion, one of the commonly used methods for recording the neurological signals is via EEG, a non-invasive method for recording the activity of large groups of neurons. In this study we performed the EEG measurements from healthy subjects performing discrete spontaneous motion (flexion and extension of the arm), and we presented a feasibility study of EEG analysis based on the temporal correlation of the brain activity.

\section{EXPERIMENTAL METHODS}

\section{A. Experimental procedure}

The experimental procedure was specified as follows;

1) After placing the electrodes on seven scalp sites over the somatosensory cortex, the subjects were asked to relax for a few minutes.

2) The subjects were asked to flex and extend the right upper limb spontaneously a few times during $60 \mathrm{~s}$.

3) After the task period subjects were asked to rest for two minutes (one trial).

4) The task and the rest period repeated 3 times, which completes one trial.

5) The subjects were asked to perform 3 sets.

The time development of the angle of the elbow was measured using a motion detection arm; the wrist was attached to the end of the motion detection arm at which the rotation axis coinsides with that of the upper limb, being measured by the rotation encoder. The ethical committee at Ritsumeikan University approved our research and the task procedure. Three healthy subjects participated in the EEG measurements following informed consent. The subjects were in their early twenties, all male, and right handed.

\section{B. EEG analysis}

EEG signals were recorded using $\mathrm{Ag}-\mathrm{AgCl}$ electrodes from seven scalp sites (international 10-20 system, F3, Fz, $\mathrm{F} 4, \mathrm{C} 3, \mathrm{Cz}, \mathrm{C} 4, \mathrm{Pz}$ ), the reference electrode was placed on the right ear (A2), and the ground was taken on the left ear (A1). Sampling frequency was set to $1 \mathrm{kHz}$. The continuous EEG signals were recorded by a multi-telemeter system (WEB-5000, Nihon Koden). The offline EEG analysis was performed as follows;

1) The time development of EEG was filtered using a band-pass filter which passed frequencies from $0.5 \mathrm{~Hz}$ to $40 \mathrm{~Hz}$. 
2) The time window was set to $1000 \mathrm{~ms}$, being shifted by $50 \mathrm{~ms}$ or $100 \mathrm{~ms}$, and the auto-correlation was calculated in each time window.

3) The zero-crossing time was defined as the time when the auto-correlation function curve intersected at $\mathrm{x}$-axis for the first time.

4) The zero-crossing time was extracted in each time window, and was plotted as a function of time for the single trials $(60 \mathrm{~s})$.

\section{RESUltS AND DiscusSiON}

The feasibility study investigated the time development of the EEG signal as subjects spontaneously flexed and extended the upper limb. Fig. 1 shows a typical example of the zero-crossing time and the single motion of the upper limb as a function of time from the electrode on Fz when the time window was shifted by $50 \mathrm{~ms}$. We observed that prior to the spontaneous motion the zero-crossing time abruptly increased and sustained the higher values by the end of the flexion motion (Fig. 1), which indicated that the correlation time of the brain activity increased. Thus, the activity ratio between the Mu rhythm (cycle time of around $100 \mathrm{~ms}$ ) and the brain activity in lower frequency region started to change prior to the movement onset.

Fig. 2 shows another example of the zero-crossing time and the single motion of the upper limb as a function of time from the electrode on F3, when the time window was shifted by $100 \mathrm{~ms}$. During $60 \mathrm{~s}$ subject A performed an elbow flexion movement 5 times and the increase of zero-crossing time was observed prior to the onset of each motion. The other two subjects basically showed the same trend. To calculate the detection rate of the motor command generation, a threshold in zero-crossing time and a persistence time above the threshold should be introduced. However, these values should be tuned for each set of experiment, which is within our scope.

Kornhuber and Deecke [4] found that a simple voluntary act such as pressing a key was preceded by an EEG component known as the "readiness potential" that began 500 $\mathrm{ms}$ to $1000 \mathrm{~ms}$ before the action. According to the gating model [5] thalamocortical loops provide a specific gating mechanism regulating the flow of signals from subcortical structures to sensory-motor corticies. The model predicts a close relationship between readiness potential and ERD in a voluntary movement in spite of differential location. The investigation of the relationship of the findings reported here to readiness potential and ERD will help to understand better the relative roles both processes play in movement preparation and will be a subject of future studies.

\section{CONCLUSION}

We presented a feasiblity study for EEG analysis based on the temporal correlation of the brain activity, which could indicate the onset of motor command generation for a single motion. The present study was based on a hypothesis that cognitive processing related to movement preparation requires coherent reorganisation of activity in various motor

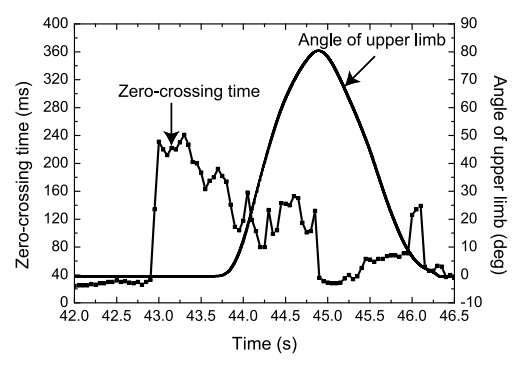

Fig. 1. The zero-crossing time and the angle of the upper limb as a function of time for subject A from the electrode on Fz. The time window was shifted by $50 \mathrm{~ms}$.

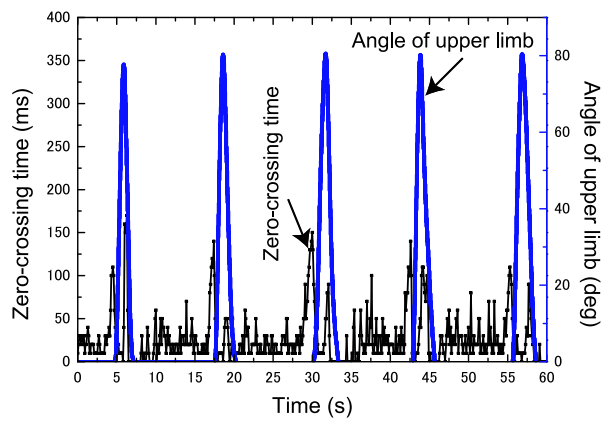

Fig. 2. The zero-crossing time and the angle of the upper limb as a function of time for subject A from the electrode on F3. The time window was shifted by $100 \mathrm{~ms}$.

cortical areas, consistent with increasingly recognised role of synchronisation in cognitive processes. The specific method of detection of changes in cortical dependencies proposed in this paper is novel and based on linear correlation. The validity of this proposed method should be checked statistically by increasing the number of trials and subjects. In the future study it would be necessary to have a certain period of bio-feedback learning, i.e., subjects might learn successful motor commands using the present EEG analysis algorithm using the actual feedback signal generated from passive motion under robotic movement guidance.

\section{REFERENCES}

[1] M. Takahashi, M. Gouko and K. Ito: "Fundamental research about electroencephalogram (EEG) - functional electrical stimulation (FES) rehabilitation system", Proc. of IEEE Int. Conf. on Robotics and Automation, 2009.

[2] R.C.V. Loureiro, W.S. Harwin, K. Nagai and M. Johnson: "Advances in upper limb stroke rehabilitation: a technology push", Med. Biol. Eng. Comput., Vol. 49, pp. 1103-1118, 2011.

[3] Y. Hayashi, K. Nagai, K. Ito, J. S. Nasuto, C.V. R. Loureiro, and S. W. Harwin: "A Feasible Study of EEG-driven Assistive Robotic System for Stroke Rehabilitation", Proc. of IEEE Int. Conf. on Biomedical Robotics and Biomechatronics, pp. 1733-1739, 2012.

[4] H.H. Kornhuber and L. Deecke: "Readiness for movement? The Bereitschaftspotential-Story", Current Contents Life Sciences 33, 4 Citation Classics January 22, 1990.

[5] M. C.M. Bastiaansena, K. B.E. Böcker, P. J.M. Cluitmansc, C. H.M. Bruniab: "Event-related desynchronization related to the anticipation of a stimulus providing knowledge of results", Clinical Neurophysiology, Vol. 110, pp. 250-260, 1999. 